\title{
In vitro Comparison of Accuracy of Two Scanners Used in Dental Medicine
}

\begin{abstract}
ALEXANDRU VICTOR BURDE ${ }^{1}$, MARIUS MANOLE ${ }^{2 *}$, RADU SEPTIMIU CAMPIAN², COSMIN SINESCU ${ }^{3}$, SORANA BACIU ${ }^{1}$
${ }^{1}$ Iuliu Hatieganu University, Faculty of Dental Medicine, Department of Dental Propaedeutics and Esthetics, 32 Clinicilor Str., 400006, Cluj Napoca, Romania

${ }^{2}$ Iuliu Hatieganu University, Faculty of Dental Medicine, Department of Oral Rehabilitation, 15 Victor Babes Str., 400012, Cluj Napoca, Romania

${ }^{3}$ Victor Babes University of Medicine and Pharmacy, Department of Dental Propaedeutics, 9 Revolutiei 1989 Blvd., 300070, Timisoara, Romania

This experimental study aims to highlight and compare the resolution and accuracy (trueness and fidelity) of an intraoral scanner and a laboratory scanner. In order to achieve the aim of this study, an experimental model consisting of a die with a standard preparation for a porcelain-fused to metal crown was manufactured from a physiognomic polymethylmethacrylate resin. The experimental model was scanned with an industrial CT to obtain the virtual reference model. Then, the die was scanned 15 times with each scanner. In order to asses trueness, comparisons were made with the virtual reference model, and for fidelity, the first scan in the series was compared with the following scans. In regards to the trueness of the scanners, the average deviation for the laboratory scanner is 4.31 and $17.34 \mu \mathrm{m}$ for the intraoral scanner. When comparing the serial scannings' of the same die, the lowest recorded deviation is $6.4 \mu \mathrm{m}$ for the laboratory scanner and $21.57 \mu \mathrm{m}$ for the intraoral scanner. For both fidelity and trueness, the $F$ test demonstrates that there is a statistically significant difference between the scanners and the applied T-tests confirm the lower degree of error-rate generation by the laboratory scanner. The dimensional limitations imposed for the intraoral scanners have a direct consequence on their trueness and fidelity when compared to their laboratory counterparts.
\end{abstract}

Keywords: CAD/CAM, scanning, accuracy, computer tomography, precision, trueness

Due to the low tensile strength of the gypsum that causes susceptibility to fracture of plaster models, along with the reduced abrasion resistance of the gypsum [1,2] and the relatively large space required for archival of plaster models [3], long-term storage of dental models may become problematic and inconvenient for dental healthcare practitioners [4]. A solution to solve these inconveniences is to digitalize the plaster models, store them in a virtual environment, and obtain physical copies of these models, if needed, through rapid prototyping systems [5-7]. Thus, in order to introduce this technology into everyday practice, the first step is to obtain the virtual model of the patient. The patient's virtual model can be obtained either by directly digitizing the dental arches through the use of optoelectronic intraoral scanners or by digitizing the conventional plaster models through indirect, laboratory scanners [8].

The first intraoral scanner, designed in 1985 by Professor Mormann and Professor Duret, was used to scan the prepared tooth and neighboring teeth [9], but intraoral scanning gradually allowed the digitization of a wider area, allowing for the scanning of a complete arch, either by capturing a series of images of the oral anatomy or by capturing video [10]. Laboratory scanners use 3 main technologies: contact scanning, structured light, or laser beam [11]. Contact scanners use a touch probe to digitize the surface of the model, and are considered to be highly accurate because they are not influenced by the optical properties of the scanned object, but these scanners are slow and may damage the plaster models. Laser beam scanners and structured light scanners use a non-contact method to digitize plaster models or conventional impressions using the active triangulation method. These scanners are faster than contact scanners, but are affected by the optical properties of the scanned material and require the application of a titanium dioxide powder or spray in order to prevent surface glare, similar to intraoral scanners[ [12]. Although intraoral scanners directly capture the topography of dental arches, eliminating the need for a

\footnotetext{
*email: mnole22@yahoo.com
}

conventional impression and/or plaster cast, and thus eliminating the errors induced by these clinical and technical steps, the accuracy of the digital models generated with these scanners may be altered by the complex factors in the oral environment, such as optical properties of teeth, saliva, or the presence of bleeding, but also by the lower resolution of these scanners compared to their laboratory counterparts[13].

In order to create a virtual model that is adequate for use in CAD/CAM reconstructions, an increased accuracy of the scanner is required. Accuracy is described by the ISO standard 5725-1 through the use of two parameters: trueness and precision. Trueness refers to the existing deviation between the actual dimension of the measured object and the measurement result itself, while precision refers to how tightly repeated measurements are. Currently, the results of several studies focused on the accuracy of intraoral and laboratory scanners are contradictory $[11,14,15]$. Although there are studies reporting a higher accuracy of intraoral scanners compared to laboratory scanners $[15,16]$, the results of these studies are difficult to compare and synthesize because there is no standard protocol for making comparisons between intraoral and laboratory scanners. In order to carry out these in vitro studies, each author uses a reference model that has different configurations, and the digital reference models used to perform the evaluation of accuracy is acquired by various digitization methods, such as: industrial structured light scanners [15,17,18], Industrial CT [19], CBCT [20,21] or coordinated measuring machines [11].

This experimental study aims to highlight and compare the resolution and accuracy (trueness and precision) of an intraoral scanner and a laboratory scanner, by using a simple reference model and industrial CT in order to perform the evaluation.

\section{Experimental part \\ Materials and method}

In order to achieve the aim of this study, an experimental model was created by using an ANA 4-Z (Frasaco Gmbh, 
Tettnang, Germany) model of a permanent maxillary right first molar which was prepared for a porcelain fused to metal crown following the standard preparation principles: $1.5 \mathrm{~mm}$ axial and proximal surface reduction, $6^{\circ}$ occlusal convergence, $2 \mathrm{~mm}$ occlusal reduction, $0.8 \mathrm{~mm}$ rounded chamfer margin. Following the preparation, the resulting die was digitized using a Dental Wings 7 Series (Dental Wings, Montreal, Canada) laboratory scanner, and the resulting mesh was optimized for CNC milling using the Meshmixer (Autodesk Inc., San Rafael, USA) software. The resulting STL file was imported in the Sum3D (CIMsystem, Cinisello Balsamo, Italy) CAM software and the milling strategy was calculated. The die was manufactured out of CopraTemp (Whitepeaks Dental Solutions GmbH \& amp; Co., Wesel, Germany) tooth-colored polymethylmethacrylate disk using a D5 (Datron AG, Mühltal Germany) CNC milling machine. We chose this material because it simulates the optical properties of the teeth to some extent, without having a high degree of translucency and brightness, properties that would interfere with structured light scanning.

After the experimental die was milled, industrial CT scanning was performed in order to obtain the virtual reference model. For this purpose, scanning was performed by using a XT H225 ST (Nikon Metrology Inc., Brighton, SUA) industrial CT scanner, at 155kV, 175 $\mathrm{AA}, 707$ ms per exposure, 1500 exposures, and 2 frames per exposure, using a $0.95 \mathrm{~mm} \mathrm{Cu}$ filter and a voxel size of 16.6 $\mathrm{m}$. The total scanning time for the reference model was 12 min and the Inspect $X$ software (Nikon Metrology Inc., Brighton, USA) was used to reconstruct the model. The resulting STL dataset was exported and defined as the reference value (REF) for this study.

For this comparison, we decided to use two non-contact scanners from the same company: a direct, chairside scanner- Apollo DI (Sirona Gmbh, Bad Sackingen, Germany) which is based on the principle of active wavefront sampling with structured light projection and allows data capturing in a video sequence, but requires light pow der dusting of the dental arch prior to scanning, and an indirect, laboratory scanner- InEos X5 (Sirona Gmbh, Bad Sackingen, Germany) which is based on the principle of optical triangulation with blue, structured light projection. With each of the two scanners, the experimental PMMA model was scanned 15 times and a STL model was generated after each scan. All scans were performed by the same operator following the manufacturer's recommended scan indications and strategies.

To determine the precision of a scanner, one of the 15 virtual models generated by a scanner was randomly chosen as a reference and was then compared to the other 14 remaining models using the best-fit superimposition algorithm of the Geomagics Qualify 2013 (Materialise, Leuven, Belgium) metrology software (fig. 1-A). To verify the trueness of the two scanners, all 15 virtual models generated by each of the two scanners were compared to virtual reference model acquired with the industrial $\mathrm{CT}$, using the same metrology software (fig. 1-B).

For each comparison, the software generated a color coded map of the registered deviations and the Root Mean Square (RMS) error of the deviations was calculated. Statistical analysis was performed with SPSS 20.0 (IBM SPSS Inc). The Levene test was used to test the homogeneity of variances and Student's t-test was used to evaluate the difference between the two groups. The level of significance was set at 0.01 .

\section{Results and discussions}

The results (mean \pm standard deviation RMS, median and interquartile amplitude) of the measurements performed using the experimental model for trueness is $21.58 \pm 5.41 \mu \mathrm{m}$ for the Apollo Di intraoral scanner. By comparison, the InEos X5 laboratory scanner was

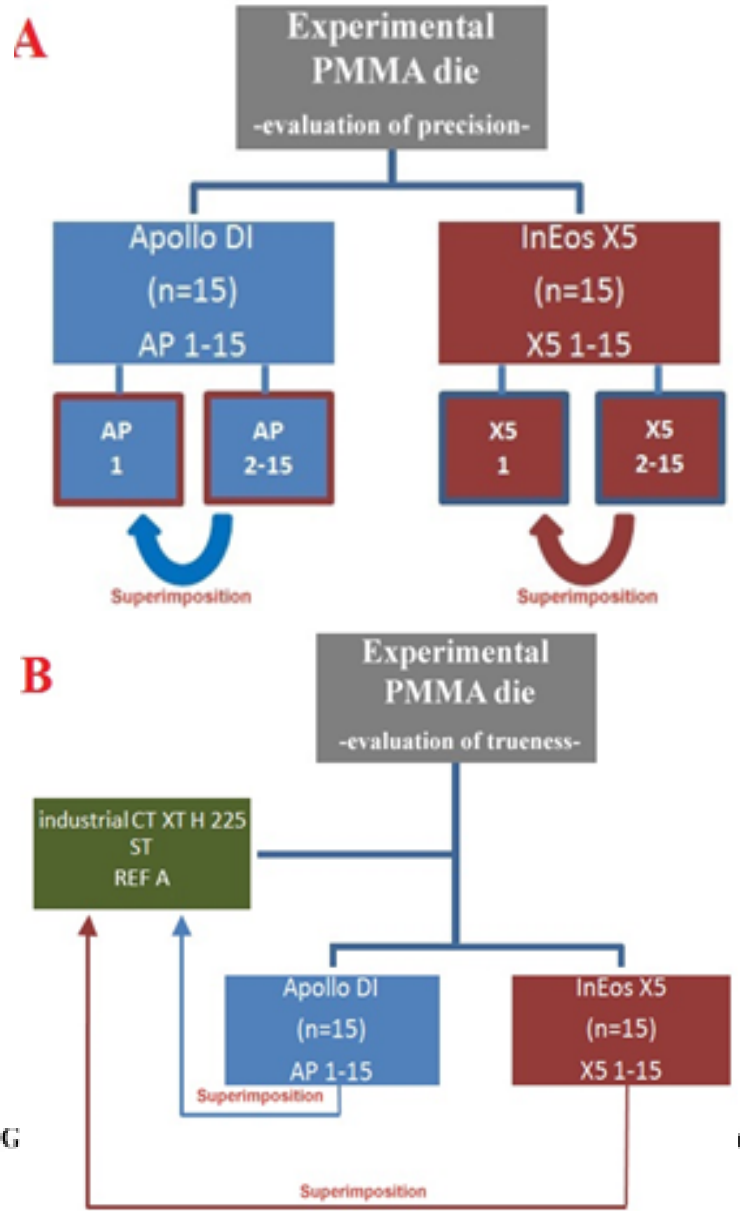

Fig. 1. Methodology used to calculate precision (A) and trueness (B)

associated with a numerically smaller mean of $6.40 \pm$ $0.55 \mu \mathrm{m}$. To test the hypothesis that the intraoral scanner and the laboratory scanner were associated with statistically significant different means, an independent samples t-test was performed. The assumption of homogeneity of variances was tested and satisfied via Levene's F test, $F(28)=53.52, p=0.000$. The independent samples t-test was associated with a statistically significant effect, $t(28)=10.85, p<0.001$. Thus, the Apollo Dl scanner was associated with a statistically significant smaller degree of trueness in comparison with the laboratory scanner. Cohen's $d$ for trueness was estimated at 3.94, which is a large effect based on Cohen's guidelines.

For precision, the results of the of the measurements performed are $17.34 \pm 2.43 \mu \mathrm{m}$ for the Apollo Di scanner and $4.32 \pm 0.34 \mu \mathrm{m}$ for the InEos X5 laboratory scanner. To test the hypothesis that the intraoral scanner and the laboratory scanner were associated with statistically significant different means for precision, an independent samples t-test was performed. The assumption of homogeneity of variances was tested and satisfied via Levene's $F$ test, $F(26)=12.33, p=0.002$. The independent samples t-test was associated with a statistically significant effect, $t(26)=19.86, p<0.001$. Thus, the InEos X5 scanner was associated with a statistically significant larger degree of precision in comparison with the intraoral scanner. Cohen's $d$ for trueness was estimated at 7.50, which is a large effect based on Cohen's guidelines.

Based on the performed tests, we can state that both in terms of precision of the two scanners and their trueness, the InEos X5 laboratory scanner has achieved better results. This could be confirmed both visually, by the high degree of overlapping in the comparison of scanned dies with the reference virtual model (fig. 2), and statistically by a very low value of $p(<0.001)$ when applying the ttests.

The CAD / CAM system has been described not as a unitary system, but as a set of distinct stages: digitization, 


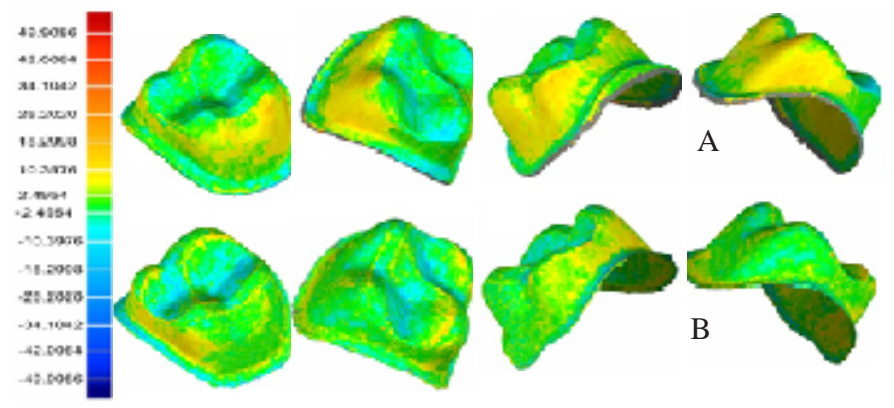

Fig. 2. Colored maps indicating deviation from the true value of the virtual reference model: A) Apollo DI, B) InEos X5

processing and manufacturing. In this case, distinct steps do not mean separate steps. These steps, even though they each have a particular specificity and need different tool to be performed, they are linked to each other as a whole. And as with a physical chain, the power of the whole chain lies in its weakest link. In this case, the first link in the step chain that forms a CAD/CAM system, namely the digitization stage, was analyzed. Despite all the advantages that the digitization has, there is a large disadvantage which is inherited from the classic impression: an improper impression will generate an incorrect model, an incorrect virtual model and eventually an inappropriate milled restoration. Despite all the progress made in the field of optoelectronics, this shortcoming of laboratory scanners will remain

The level of precision that dental restorations require has increased nowadays. The support provided by dental implants requires total passivity of the restoration that is applied to this interface. And, in order to manufacture an implant restoration in this appropriate manner, the impression must contain a minimum number of errors. The increased level of precision brings about a number of problems. In the case of conventional impressions, errors could be more easily identified because of elements such as: the amount of impression material, its quality, and how the prosthetic field was recorded. In the case of optoelectronic impressions, the impression errors are in the order of microns, and checking for these errors is more difficult. Moreover, the digital model is composed of a series of multiple images that the software algorithm stiches to form a three-dimensional object. The way in which this algorithm operates can be programmed, but cannot be guaranteed that it will alw ays make the best frame overlap with the scanned object. Thus, optoelectronic scanning brings with it a multitude of advantages that cannot be denied, but at the same time raises a number of unique problems that cannot be ignored.

The present study focused on the digitization procedure in its two variants, the laboratory version and the chairside version. Differences between scanners are not related only on the mode they are used, but they are related to the environment and the procedure where the digitization takes place. The results have demonstrated increased precision and trueness of the In Eos X5 laboratoryscanner. But the scanning protocol used for this scanner was automated. The operator does not affect the scanning procedure in anyway. The intraoral scanner used in the study, Apollo DI, had a full manual scanning protocol. Also, a laboratory scanner has no limitto the space it can occupy. However, the chair-side scanner musthave a small volume so that it can be handled in the oral cavity of the patient without causing discomfort. The lab scanner usually digitizes a gypsum model or an impression, while the chairside scanner operates in a wetenvironment, in which saliva and the optical proprieties of teeth influence the scanning procedure.

\section{Conclusions}

Given the fact that the test conditions used in this invitro study are not correlated with the use of these scanners in the clinical, daily practice, the results of this study do not represent a clear victory of the laboratory scanner against the chair-side scanner, but represent a state in which the laboratory scanner provided increased accuracy. However, several conclusions can be drawn:

The laboratory scanner showed a small degree of dimensional errors in the generated digital models, both in terms of precision and trueness.

Errors displayed by the laboratory scanner have a low degree of variability, which opens the possibility that they can be compensated for in later stages of the CAD / CAM procedure.

The chair-side scanner has shown a high degree of variability in recorded dimensional errors, but a large proportion of the scanning protocol is entirely manual.

The laboratory scanner is greatly enhanced by the high degree of mobility in the scanning process, as well as the ability to scan models with removable dies. At the same time, the laboratory scanner is disadvantaged by the fact that it digitizes conventional models or impressions, which already passed through several clinical and technical steps, which could affect their real dimensions.

\section{References}

1. NICOLA C. Materiale dentare- consideraii clinice si tehnice.Editura Casa Cartii de Stiita, Cluj-Napoca, 2009, p. 94.

2. DUKE P., MOORE K., HAUG S., ANDRES C., J Prosthet Dent., 83, no.4, 2000, p. 466.

3. MCGUINNESS, NJ ., STEPHENS, CD., Br J Orthod., 19, no.3, 1992, p. 227.

4. KEATING, P., PARKER, RA., KEANE, D., WRIGHT, L., Br J Orthod., 11, no. 3, 1984, p.119.

5. KASPAROVA, MM., GRAFOVA, L., DVORAK, P., DOSTALOVA, T., PROCHAZKA, A., ELIASOVA, H., Biomed Eng Online.,12, no.1, 2013, p.49.

6. VAN NOORT, R., Dent Mater., 28, no.1,2012, p.3.

7. HAZEVELD, A., HUDDLESTON, JJ R., REN, Y., Am J Orthod Dentofac Orthop., 145, no.1, 2014, p. 108.

8. UZUN, G.,Biotechnol Biotechnol Equip., 22, no.1, 2008, p.530.

9. DURET, F., BLOUIN, JL., DURET, B., J Am Dent Assoc., 117, no.6, 1988, p.715.

10. DAVIDOWITZ, G., KOTICK, PG., Dent Clin North Am., 55, no.3, 2011,p.559.

11.GONZALEZ DE VILLAUMBROSIA, P., MARTINEZ-RUS, F., GARCIAOREJAS, A., SALIDO, MP., PRADIES, G., J Prosthet Dent., 116, no. 4, 2016, p. 543

12.BURDE, A.V.., DUDEA, D., CUC, S., MOLDOVAN, M., CAMPIAN, R.S., Mat. Plast., 53, no. 1, 2016, p. 65.

13. TODOROVIC, A., LAZIC, V., Stomatol Glas Srb., 53, no.1, 2006, p. 42.

14.MANDELLI, F., GHERLONE, E., GASTALDI, G., FERRARI, M., JProsthodont Res., 60, no. 1, 2016, p. 2.

15. PATZELT, SBM., EMMANOUILIDI, A., STAMPF, S., STRUB, JR., ATT, W., Clin Oral Investig., 18, no.6, 2014, p. 1687.

16. RENNE, W., LUDLOW, M., FRYML, J., SCHURCH, Z., MENNITO, A.,KESSLER, R., J Prosthet Dent., 118, no. 1, 2016, p. 36.

17. JEON, JH., KIM, HY., KIM, J H., KIM, WC., J Adv Prosthodont.,6, no. 6, 2014, p.468.

18. NEDELCU, RG., PERSSON, ASK., J Prosthet Dent., 112, no.6, 2014, p.1461.

19. GÜTH, JF., KEUL, C., STIMMELMAYR, M., BEUER, F., EDELHOFF, D., Clin Oral Investig., 17, no. 4, 2013, p.1201.

20. KIM, J., HEO, G., LAGRAVERE, MO., Angle Orthod., 84, no.3, 2014, p. 443. A

21. AKYALCIN, S., DYER, DJ., ENGLISH, JD., SAR, C.,Am J Orthod Dentofacial Orthop., 144, no. 6, 2013,p. 831. 